Supplement of Biogeosciences Discuss., 11, 8569-8605, 2014

http://www.biogeosciences-discuss.net/bgd-11-8569-2014/

doi:10.5194/bgd-11-8569-2014-supplement

(C) Author(s) 2014. CC Attribution 3.0 License.

(c) (i)

Supplement of

\title{
Forms of organic phosphorus in wetland soils
}

A. W. Cheesman et al.

Correspondence to: A. W. Cheesman (alexander.cheesman@gmail.com) 
Supplement of Biogeosciences Discuss., 11, 8569-8605, 2014

http://www.biogeosciences-discuss.net/bgd-11-8569-2014/

doi:10.5194/bgd-11-8569-2014-supplement

(C) Author(s) 2014. CC Attribution 3.0 License.

(c) (i)

Supplement of

\section{Forms of organic phosphorus in wetland soils}

A. W. Cheesman et al.

Correspondence to: A. W. Cheesman (alexander.cheesman@gmail.com) 
Supplementary Table 1 . Twenty eight wetland sites sampled for characterization of phosphorus composition.

\begin{tabular}{|c|c|c|c|c|c|c|}
\hline & Wetland & Location & Wetland Type $^{\dagger}$ & Vegetation Type & Dominant species ${ }^{\S}$ & $\begin{array}{l}\text { Potential } \\
\text { impacts }\end{array}$ \\
\hline 1 & 8 mile & $\mathrm{Al}, \mathrm{USA}$ & Bog & $\begin{array}{l}\text { Persistent emergent } \\
\text { (Moss) }\end{array}$ & Sphagnum, Carex sp. & \\
\hline 2 & Laguna Papallacta, & Ecuador & $\begin{array}{l}\text { Cushion } \\
\text { forming, } \\
\text { Paramo }\end{array}$ & $\begin{array}{l}\text { Persistent emergent } \\
\text { (Herbaceous) }\end{array}$ & $\begin{array}{l}\text { Self-emergent succulent species Distichia muscoides, } \\
\text { Spagnum }\end{array}$ & Cattle Grazing \\
\hline 3 & Wicken Fen & UK & Fen & $\begin{array}{l}\text { Persistent emergent } \\
\text { (Herbaceous) }\end{array}$ & Cladium mariscus & $\begin{array}{l}\text { Sedge } \\
\text { Harvesting } \\
\text { since } 1419\end{array}$ \\
\hline 4 & $\begin{array}{l}\text { Houghton lake } \\
\text { (CT350) }\end{array}$ & MI, USA & $\begin{array}{l}\text { Treatment } \\
\text { wetland }\end{array}$ & $\begin{array}{l}\text { Persistent emergent } \\
\text { (Herbaceous) }\end{array}$ & Typha sp. & $\begin{array}{l}\text { Intermediate P } \\
\text { loading }\end{array}$ \\
\hline 5 & Houghton lake (550C) & MI, USA & $\begin{array}{l}\text { Treatment } \\
\text { wetland }\end{array}$ & $\begin{array}{l}\text { Persistent emergent } \\
\text { (Herbaceous) }\end{array}$ & Cyperacea sp. Typha & Low P loading \\
\hline 6 & Houghton lake (P) & MI, USA & $\begin{array}{l}\text { Treatment } \\
\text { wetland }\end{array}$ & $\begin{array}{l}\text { Persistent emergent } \\
\text { (Herbaceous) }\end{array}$ & Typha. & High P loading \\
\hline 7 & $\begin{array}{l}\text { Francis Marion } \\
\text { National Forest } \\
(\text { FMNF) Bay } 1\end{array}$ & SC,USA & Carolina Bay & $\begin{array}{l}\text { Persistent emergent } \\
\text { (Forested) }\end{array}$ & $\begin{array}{l}\text { Acer rubrum (var. trilobum), Nyssa biflora and Nyssa } \\
\text { aquatica, Lyonia lucida, Ilex myrtifolia }\end{array}$ & \\
\hline 8 & $\begin{array}{l}\text { Francis Marion } \\
\text { National Forest } \\
(\text { FMNF) Bay } 2\end{array}$ & SC,USA & Carolina Bay & $\begin{array}{l}\text { Persistent emergent } \\
\text { (Forested) }\end{array}$ & $\begin{array}{l}\text { Taxodium ascendans, Nyssa biflora, Lyonia lucida, Carex } \\
\text { striata, Woodwardia virginica }\end{array}$ & \\
\hline 9 & $\begin{array}{l}\text { Francis Marion } \\
\text { National Forest } \\
(\text { FMNF) Bay } 3\end{array}$ & SC,USA & Carolina Bay & $\begin{array}{l}\text { Persistent emergent } \\
\text { (Herbaceous) }\end{array}$ & $\begin{array}{l}\text { Ilex glabra, Iris tridentata, Amphicarpum } \\
\text { muhlenbergianum, Eleocharis spp., Melanocarpa, } \\
\text { tricostata, and Lachnanthes caroliniana }\end{array}$ & $\begin{array}{l}\text { Periodic } \\
\text { burning }\end{array}$ \\
\hline 10 & $\begin{array}{l}\text { Francis Marion } \\
\text { National Forest } \\
(\text { FMNF) Bay } 4\end{array}$ & SC,USA & Carolina Bay & $\begin{array}{l}\text { Persistent emergent } \\
\text { (Forested) }\end{array}$ & $\begin{array}{l}\text { Nyssa biflora, Taxodium ascendans, Acer rubrum, Lyonia } \\
\text { lucida, Cyrilla racemiflora, Pinus taeda }\end{array}$ & \\
\hline 11 & $\begin{array}{l}\text { Savannah River Site } \\
\text { (SRS) Bay } 1\end{array}$ & SC,USA & Carolina Bay & $\begin{array}{l}\text { Persistent emergent } \\
\text { (forested/herbaceous) }\end{array}$ & $\begin{array}{l}\text { Panicum hemitomon, Nyssa biflora, Cephalanthus } \\
\text { occidentalis, Utricularia spp., Sphagnum spp., Pontederia } \\
\text { cordata var. lancifolia }\end{array}$ & \\
\hline 12 & $\begin{array}{l}\text { Savannah River Site } \\
\text { (SRS) Bay } 2\end{array}$ & SC,USA & Carolina Bay & $\begin{array}{l}\text { Persistent emergent } \\
\text { (Herbaceous) }\end{array}$ & $\begin{array}{l}\text { Panicum hemitomon, Sphagnum spp., Pontederia cordata } \\
\text { var. lancifolia, Juncus canadensis, Cephalanthus } \\
\text { occidentalis, Acer rubrum (var. trilobum) }\end{array}$ & \\
\hline 13 & $\begin{array}{l}\text { Savannah River Site } \\
\text { (SRS) Bay } 3\end{array}$ & SC,USA & Carolina Bay & Open water /herbaceous & $\begin{array}{l}\text { Nymphaea odorata, Panicum hemitomon, Utricularia } \\
\text { spp., Leersia hexandra, Eleocharis melanocarpa }\end{array}$ & \\
\hline 14 & $\begin{array}{l}\text { Svannah River Site } \\
\text { (SRS) Bay } 4\end{array}$ & SC,USA & Carolina Bay & $\begin{array}{l}\text { Persistent emergent } \\
\text { (Forested) }\end{array}$ & $\begin{array}{l}\text { Liquidambar styraciflua, Acer rubrum (var. trilobum), } \\
\text { Nyssa biflora, Taxodium ascendans, Smilax rotundifolia }\end{array}$ & \\
\hline 15 & Larry Fen & NY, USA & Rich Fen & Persistent emergent & Carex sp. Campylium stellatum & \\
\hline
\end{tabular}




\begin{tabular}{|c|c|c|c|c|c|c|}
\hline 16 & Fish Fen & NY, USA & Rich Fen & $\begin{array}{l}\text { (Herbaceous) } \\
\text { Persistent emergent } \\
\text { (Herbaceous) }\end{array}$ & $\begin{array}{l}\text { Typha angustifolia. Carex sp. Campylium stellatum, } \\
\text { Sphagnum spp.Calliergonella cuspidata }\end{array}$ & \\
\hline 17 & Hidden & Belize & $\begin{array}{l}\text { Oligotrophic } \\
\text { Sumpland }\end{array}$ & $\begin{array}{l}\text { Persistent emergent } \\
\text { (Herbaceous/ } \\
\text { Cyanobacteria) }\end{array}$ & Eleocharis cellulosa, Cyanobacteria spp. & salt intrusion \\
\hline 18 & Quiet & Belize & Sumpland & $\begin{array}{l}\text { Persistent emergent } \\
\text { (Herbaceous/ } \\
\text { Cyanobacteria) }\end{array}$ & Eleocharis cellulosa, Cyanobacteria spp. & salt intrusion \\
\hline 19 & Doubloon & Belize & Sumpland & $\begin{array}{l}\text { Persistent emergent } \\
\text { (Herbaceous/ } \\
\text { Cyanobacteria) }\end{array}$ & Eleocharis cellulosa, Cyanobacteria spp. & salt intrusion \\
\hline 20 & Changuinola Site 1 & Panama & $\begin{array}{l}\text { Tropical peat } \\
\text { dome }\end{array}$ & $\begin{array}{l}\text { Persistent emergent } \\
\text { (Forested) }\end{array}$ & Raphia tadeiga & \\
\hline 21 & Changuinola Site 2 & Panama & $\begin{array}{l}\text { Tropical peat } \\
\text { dome }\end{array}$ & $\begin{array}{l}\text { Persistent emergent } \\
\text { (Forested) }\end{array}$ & $\begin{array}{l}\text { Campnosperma panamensis, Cassipourea elliptica (Sw.) } \\
\text { Poir, Drypetes standleyi G.L. Webster }\end{array}$ & \\
\hline 22 & Changuinola Site 3 & Panama & $\begin{array}{l}\text { Tropical peat } \\
\text { dome }\end{array}$ & $\begin{array}{l}\text { Persistent emergent } \\
\text { (Forested) }\end{array}$ & $\begin{array}{l}\text { Campnosperma panamensis, Cyrilla racemiflora, } \\
\text { sawgrass }\end{array}$ & \\
\hline 23 & WCA 3A & Fl, USA & $\begin{array}{l}\text { Calcareous } \\
\text { Fen }\end{array}$ & Open water & Nymphaea sp. Utricularia & \\
\hline 24 & $\begin{array}{l}\text { Everglades National } \\
\text { Park }\end{array}$ & Fl, USA & $\begin{array}{l}\text { Calcareous } \\
\text { Fen }\end{array}$ & $\begin{array}{l}\text { Persistent emergent } \\
\text { (Herbaceous) }\end{array}$ & Cladium jamaicense & \\
\hline 25 & Ny Alesund & $\begin{array}{l}\text { Spitsbergen, } \\
\text { Norway }\end{array}$ & Wet tundra & $\begin{array}{l}\text { Persistent emergent } \\
\text { (Moss) }\end{array}$ & Calliergon richardsoni, Poa arctica, Dupotia species & Geese grazing \\
\hline 26 & Stordalen & $\begin{array}{l}\text { Abisko, } \\
\text { Sweden }\end{array}$ & Mire & $\begin{array}{l}\text { Persistent emergent } \\
\text { (Moss) }\end{array}$ & $\begin{array}{l}\text { Spagnum fuscum, Betula nana, Rubus } \\
\text { chamaemorus, Vaccinium vitis ideae, Empetrum nigrum }\end{array}$ & \\
\hline 27 & Bog 8 & Canada & $\begin{array}{l}\text { Ombrotrophic } \\
\text { Bog }\end{array}$ & $\begin{array}{l}\text { Persistent emergent } \\
\text { (Moss) }\end{array}$ & Sphagnum fuscum, graminoids, Lichens & \\
\hline 28 & Fen 1 & Canada & Fen & $\begin{array}{l}\text { Persistent emergent } \\
\text { (Herbaceous) }\end{array}$ & Carex sp. & \\
\hline
\end{tabular}


Supplementary Table 2. Biogeochemical properties of surface $(0-10 \mathrm{~cm})$ soils in 28 palustrine wetland systems. Values represent arithmetic mean $\pm 1 \mathrm{SD}$.

\begin{tabular}{|c|c|c|c|c|c|c|c|c|c|c|c|c|c|c|c|c|c|c|c|c|c|c|c|c|c|c|c|c|}
\hline \multirow{3}{*}{$\begin{array}{l}\text { Wetland } \\
\text { Group A }\end{array}$} & \multirow{3}{*}{$\mathrm{pH}$} & \multirow{2}{*}{\multicolumn{3}{|c|}{$\begin{array}{c}\text { Organic } \\
\text { matter } \\
\%\end{array}$}} & \multirow{2}{*}{\multicolumn{3}{|c|}{$\begin{array}{c}\text { Phosphorus } \\
\qquad \mathrm{g} \mathrm{g}^{-1}\end{array}$}} & \multirow{2}{*}{\multicolumn{3}{|c|}{ Total C }} & \multicolumn{3}{|c|}{ Total N } & \multirow{2}{*}{\multicolumn{3}{|c|}{$\begin{array}{r}\text { Total Al } \\
\mathrm{mg} \mathrm{g}^{-1}\end{array}$}} & \multirow{2}{*}{\multicolumn{3}{|c|}{ Total Ca }} & \multicolumn{3}{|c|}{ Total Fe } & \multicolumn{6}{|c|}{ Molar Ratio } \\
\hline & & & & & & & & & & & & & & & & & & & & & & & \multicolumn{3}{|c|}{$\mathrm{C}: \mathrm{P}$} & \multicolumn{3}{|c|}{$\mathrm{N}: \mathrm{P}$} \\
\hline & & & & & & & & & & & & & & & & & & & & & & & & & & & & \\
\hline 27 & 3.9 & 97 & \pm & 1 & 356 & \pm & 62 & 436 & \pm & 11 & 7.8 & \pm & 0.3 & 0.5 & \pm & 0.2 & 3.2 & \pm & 0.7 & 0.3 & \pm & 0.0 & 3220 & \pm & 493 & 49 & \pm & 8 \\
\hline 26 & 4.1 & 100 & \pm & NA & 238 & \pm & NA & 424 & \pm & NA & 6.3 & \pm & & 0.4 & \pm & NA & 1.8 & \pm & NA & 0.2 & \pm & NA & 4596 & \pm & NA & 59 & \pm & NA \\
\hline 22 & 3.7 & 84 & \pm & 5 & 579 & \pm & 102 & 424 & \pm & 30 & 21.7 & \pm & 3.1 & 1.2 & \pm & 0.2 & 1.0 & \pm & 0.1 & 1.5 & \pm & 0.4 & 1929 & \pm & 289 & 83 & \pm & 5 \\
\hline 21 & 3.6 & 93 & \pm & 1 & 852 & \pm & 130 & 485 & \pm & 12 & 25.1 & \pm & 1.8 & 1.3 & \pm & 0.1 & 3.3 & \pm & 0.5 & 3.7 & \pm & 0.6 & 1498 & \pm & 234 & 66 & \pm & 7 \\
\hline 20 & 3.8 & 88 & \pm & 1 & 1124 & \pm & 38 & 489 & \pm & 4 & 27.7 & \pm & 0.8 & 3.0 & \pm & 0.1 & 1.4 & \pm & 0.2 & 6.2 & \pm & 0.7 & 1125 & \pm & 40 & 55 & \pm & 3 \\
\hline 1 & 4.6 & 92 & \pm & 1 & 986 & \pm & 72 & 410 & \pm & 9 & 12.4 & \pm & 1.5 & 3.0 & \pm & 0.5 & 4.7 & \pm & 0.5 & 15.0 & \pm & 2.5 & 1060 & \pm & 70 & 27 & \pm & 2 \\
\hline mean & 4.0 & 92 & & & 689 & & & 445 & & & 16.8 & & & 1.6 & & & 2.6 & & & 4.5 & & & 2238 & & & 57 & & \\
\hline $\min$ & 3.6 & 84 & & & 238 & & & 410 & & & 6.3 & & & 0.4 & & & 1.0 & & & 0.2 & & & 1060 & & & 27 & & \\
\hline $\max$ & 4.6 & 100 & & & 1124 & & & 489 & & & 27.7 & & & 3.0 & & & 4.7 & & & 15.0 & & & 4596 & & & 83 & & \\
\hline \multicolumn{29}{|l|}{ Group B } \\
\hline 14 & 4.4 & 23 & \pm & 5 & 918 & \pm & 253 & 94 & \pm & 25 & 7.2 & \pm & 2.3 & 77.1 & \pm & 3.3 & 0.2 & \pm & 0.1 & 6.2 & \pm & 1.9 & 285 & \pm & 120 & 18 & \pm & 7 \\
\hline 13 & 4 & 25 & \pm & 18 & 347 & \pm & 233 & 117 & \pm & 89 & 9.3 & \pm & 6.9 & 26.2 & \pm & 6.7 & 0.2 & \pm & 0.2 & 2.9 & \pm & 0.7 & 814 & \pm & 121 & 56 & \pm & 6 \\
\hline 12 & 3.9 & 55 & \pm & 10 & 1056 & \pm & 120 & 307 & \pm & 67 & 21.4 & \pm & 4.5 & 15.7 & \pm & 4.0 & 1.2 & \pm & 0.6 & 3.7 & \pm & 0.5 & 745 & \pm & 97 & 45 & \pm & 6 \\
\hline 11 & 4.3 & 24 & \pm & 11 & 918 & \pm & 320 & 105 & \pm & 58 & 8.7 & \pm & 4.1 & 72.9 & \pm & 8.2 & 0.4 & \pm & 0.3 & 5.6 & \pm & 0.8 & 283 & \pm & 59 & 21 & \pm & 3 \\
\hline 10 & 4.4 & 48 & \pm & 18 & 752 & \pm & 422 & 239 & \pm & 115 & 12.7 & \pm & 6.0 & 34.7 & \pm & 7.6 & 0.3 & \pm & 0.3 & 4.7 & \pm & 1.1 & 1079 & \pm & 65 & 49 & \pm & 4 \\
\hline 9 & 4.2 & 9 & \pm & 4 & 51 & \pm & 35 & 44 & \pm & 16 & 2.2 & \pm & 0.8 & 2.9 & \pm & 1.5 & -0.1 & \pm & 0.1 & 0.7 & \pm & 0.3 & 2551 & \pm & 748 & 111 & \pm & 26 \\
\hline 8 & 3.5 & 69 & \pm & 21 & 750 & \pm & 165 & 376 & \pm & 131 & 19.0 & \pm & 5.3 & 8.2 & \pm & 2.6 & 2.0 & \pm & 1.3 & 2.1 & \pm & 0.5 & 1275 & \pm & 189 & 56 & \pm & 4 \\
\hline 7 & 3.6 & 50 & \pm & 17 & 925 & \pm & 169 & 260 & \pm & 87 & 14.5 & \pm & 3.6 & 25.8 & \pm & 2.0 & 0.7 & \pm & 0.4 & 3.3 & \pm & 1.6 & 729 & \pm & 191 & 35 & \pm & 6 \\
\hline mean & 4.0 & 38 & & & 715 & & & 193 & & & 11.9 & & & 32.9 & & & 0.6 & & & 3.6 & & & 970 & & & 49 & & \\
\hline $\min$ & 3.5 & 9 & & & 51 & & & 44 & & & 2.2 & & & 2.9 & & & -0.1 & & & 0.7 & & & 283 & & & 18 & & \\
\hline $\max$ & 4.4 & 69 & & & 1056 & & & 376 & & & 21.4 & & & 77.1 & & & 2.0 & & & 6.2 & & & 2551 & & & 111 & & \\
\hline \multicolumn{29}{|l|}{ Group C } \\
\hline 2 & 6.3 & 89 & \pm & 1 & 679 & \pm & 5 & 406 & \pm & 2 & 26.3 & \pm & 5.0 & 1.0 & \pm & 0.3 & 18.4 & \pm & 4.4 & 0.4 & \pm & 0.0 & 1545 & \pm & 19 & 86 & \pm & 15 \\
\hline 28 & 6.1 & 90 & \pm & 1 & 310 & \pm & 55 & 443 & \pm & 12 & 30.4 & \pm & 2.7 & 3.5 & \pm & 1.6 & 26.1 & \pm & 1.7 & 0.2 & \pm & 0.0 & 3800 & \pm & 843 & 225 & \pm & 62 \\
\hline 24 & 5.9 & 93 & \pm & 1 & 277 & \pm & 16 & 445 & \pm & 14 & 36.1 & \pm & 2.0 & 2.3 & \pm & 0.5 & 26.0 & \pm & 1.2 & 0.2 & \pm & 0.0 & 4170 & \pm & 348 & 290 & \pm & 31 \\
\hline 23 & 7 & 85 & \pm & 2 & 1184 & \pm & 138 & 421 & \pm & 17 & 28.3 & \pm & 1.3 & 3.6 & \pm & 1.4 & 31.6 & \pm & 1.5 & 4.6 & \pm & 1.6 & 929 & \pm & 120 & 53 & \pm & 6 \\
\hline 16 & 7 & 56 & \pm & 5 & 1184 & \pm & 52 & 270 & \pm & 24 & 20.3 & \pm & 1.7 & 20.4 & \pm & 4.2 & 27.3 & \pm & 2.6 & 18.8 & \pm & 1.0 & 591 & \pm & 74 & 38 & \pm & 5 \\
\hline 15 & 6.4 & 70 & \pm & 6 & 3516 & \pm & 442 & 353 & \pm & 26 & 35.2 & \pm & 2.8 & 15.2 & \pm & 3.7 & 23.0 & \pm & 5.0 & 18.9 & \pm & 5.4 & 261 & \pm & 15 & 22 & \pm & 1 \\
\hline 6 & 6.1 & 94 & \pm & 0 & 982 & \pm & 67 & 455 & \pm & 6 & 20.1 & \pm & 1.2 & 0.9 & \pm & 0.1 & 8.6 & \pm & 0.8 & 7.2 & \pm & 0.8 & 1201 & \pm & 87 & 45 & \pm & 4 \\
\hline 5 & 7.2 & 94 & \pm & 0 & 1439 & \pm & 59 & 452 & \pm & 1 & 26.2 & \pm & 0.6 & 1.0 & \pm & 0.2 & 14.0 & \pm & 1.1 & 6.0 & \pm & 0.2 & 811 & \pm & 31 & 40 & \pm & 1 \\
\hline 4 & 7.3 & 78 & \pm & 15 & 937 & \pm & 85 & 353 & \pm & 14 & 26.3 & \pm & 2.2 & 3.7 & \pm & 0.6 & 96.2 & \pm & 24.5 & 12.2 & \pm & 1.5 & 976 & \pm & 60 & 62 & \pm & 1 \\
\hline
\end{tabular}




\begin{tabular}{|c|c|c|c|c|c|c|c|c|c|c|c|c|c|c|c|c|c|c|c|c|c|c|c|c|c|c|c|c|c|}
\hline & 3 & 6.7 & 84 & \pm & 2 & 875 & \pm & 175 & 399 & \pm & 14 & 14.8 & \pm & 2.1 & 3.4 & \pm & 0.8 & 20.1 & \pm & 2.3 & 3.7 & \pm & 1.2 & 1213 & \pm & 230 & 38 & \pm & 4 \\
\hline mean & & 6.6 & 83 & & & 1138 & & & 400 & & & 26.4 & & & 5.5 & & & 29.1 & & & 7.2 & & & 1550 & & & 90 & & \\
\hline $\min$ & & 5.9 & 56 & & & 277 & & & 270 & & & 14.8 & & & 0.9 & & & 8.6 & & & 0.2 & & & 261 & & & 22 & & \\
\hline $\max$ & & 7.3 & 94 & & & 3516 & & & 455 & & & 36.1 & & & 20.4 & & & 96.2 & & & 18.9 & & & 4170 & & & 290 & & \\
\hline \multicolumn{30}{|c|}{ Group D } \\
\hline & 25 & 7 & 29 & \pm & 10 & 1513 & \pm & 773 & 133 & \pm & 57 & 5.8 & \pm & 2.0 & 19.9 & \pm & 2.5 & 15.1 & \pm & 3.3 & 0.2 & \pm & 0.0 & 247 & \pm & 114 & 9 & \pm & 3 \\
\hline & 19 & 7.6 & 16 & \pm & 3 & 126 & \pm & 25 & 153 & \pm & 0 & 5.3 & \pm & 0.1 & 2.3 & \pm & 0.7 & 333.5 & \pm & 15.4 & 0.2 & \pm & 0.0 & 3213 & \pm & 603 & 96 & \pm & 19 \\
\hline & 18 & 7.5 & 30 & \pm & 1 & 287 & \pm & 324 & 70 & \pm & 6 & 6.0 & \pm & 0.5 & 45.8 & \pm & 3.2 & 14.4 & \pm & 0.8 & 0.2 & \pm & 0.0 & 1338 & \pm & 954 & 94 & \pm & 65 \\
\hline & 17 & 7.3 & 25 & \pm & 2 & 192 & \pm & 55 & 162 & \pm & 12 & 11.4 & \pm & 0.2 & 15.3 & \pm & 7.1 & 232.3 & \pm & 51.1 & 0.2 & \pm & 0.0 & 2305 & \pm & 654 & 137 & \pm & 34 \\
\hline mean & & 7.4 & 25 & & & 530 & & & 129 & & & 7.1 & & & 20.8 & & & 148.8 & & & 0.2 & & & 1776 & & & 84 & & \\
\hline $\min$ & & 7.0 & 16 & & & 126 & & & 70 & & & 5.3 & & & 2.3 & & & 14.4 & & & 0.2 & & & 247 & & & 9 & & \\
\hline $\max$ & & 7.6 & 30 & & & 1513 & & & 162 & & & 11.4 & & & 45.8 & & & 333.5 & & & 0.2 & & & 3213 & & & 137 & & \\
\hline
\end{tabular}


Supplementary Table 3: Phosphorus composition of surface soils as determined by solution ${ }^{31} \mathrm{P}$ NMR spectroscopy. Values represent concentration $\mu \mathrm{g} \mathrm{g}^{-1}$ (\% of total soil P)

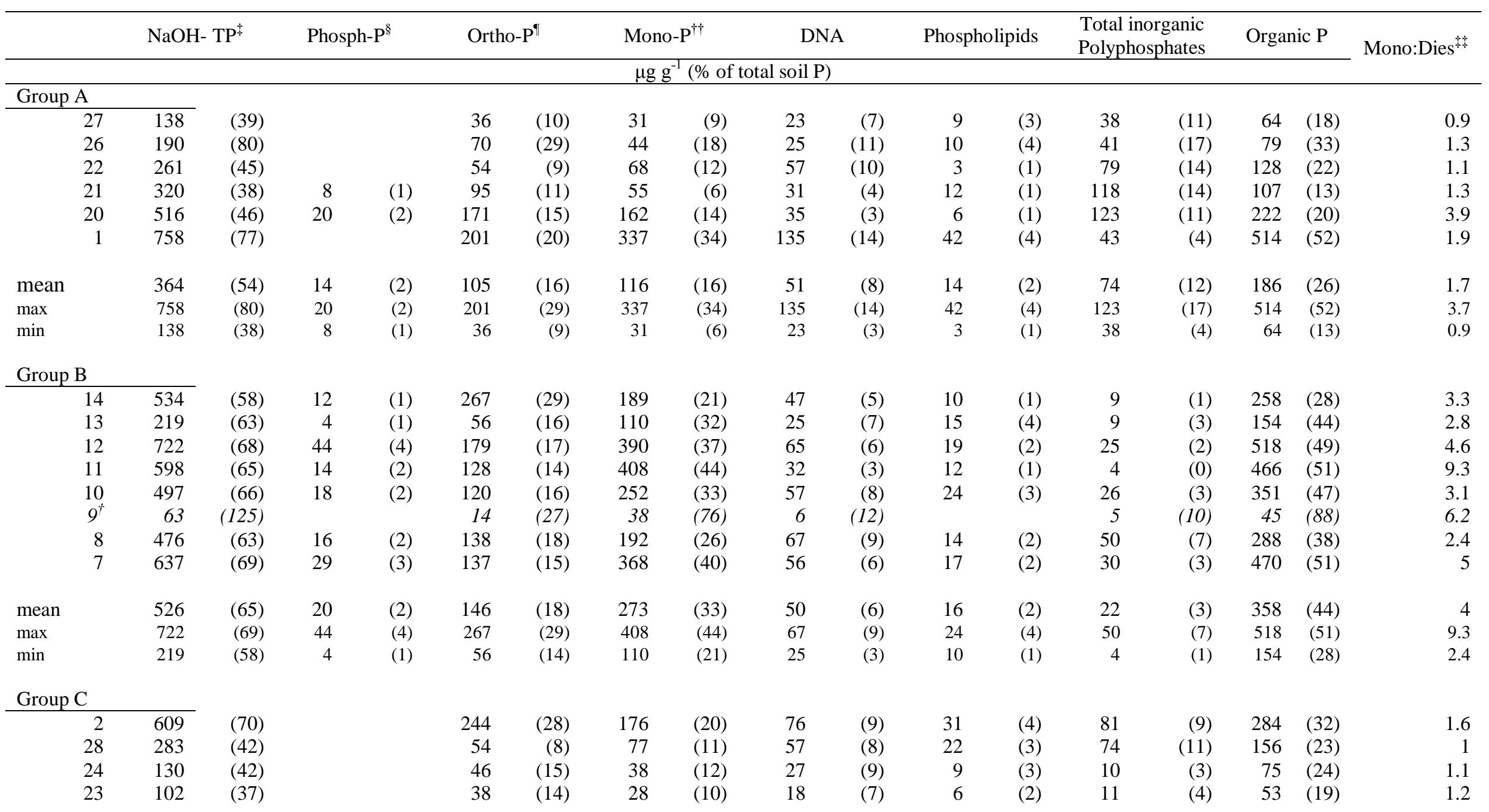




\begin{tabular}{|c|c|c|c|c|c|c|c|c|c|c|c|c|c|c|c|c|}
\hline & 16 & 595 & $(50)$ & 131 & (11) & 270 & (23) & 106 & (9) & 28 & (2) & 60 & (5) & 404 & (34) & 2 \\
\hline & 15 & 753 & (64) & 118 & (10) & 461 & (39) & 88 & (7) & 37 & (3) & 50 & (4) & 586 & (49) & 3.7 \\
\hline & 6 & 2569 & (73) & 1759 & (50) & 407 & (12) & 141 & (4) & 64 & (2) & 197 & (6) & 612 & (17) & 2 \\
\hline & 5 & 593 & (60) & 225 & (23) & 170 & (17) & 96 & (10) & 30 & (3) & 72 & (7) & 296 & (30) & 1.3 \\
\hline & 4 & 909 & (63) & 295 & (20) & 317 & (22) & 142 & (10) & 67 & (5) & 88 & (6) & 526 & (37) & 1.5 \\
\hline & 3 & 789 & (84) & 167 & (18) & 344 & (37) & 144 & (15) & 62 & (7) & 73 & (8) & 549 & (59) & 1.7 \\
\hline mean & & 733 & (59) & 308 & (20) & 229 & (20) & 90 & (9) & 36 & (3) & 72 & (6) & 354 & $(32$ & 2 \\
\hline $\max$ & & 2569 & (84) & 1759 & (50) & 461 & (39) & 144 & (15) & 67 & (7) & 197 & (11) & 612 & $(59$ & 4 \\
\hline $\min$ & & 102 & (37) & 38 & (8) & 28 & (10) & 18 & (4) & 6 & (2) & 10 & (3) & 53 & (17) & 1 \\
\hline \multicolumn{17}{|c|}{ Group D } \\
\hline & 25 & 534 & (35) & 292 & (19) & 221 & (15) & 11 & (1) & 10 & (1) & & & 242 & (16) & 10.6 \\
\hline & 19 & 33 & (26) & 33 & (26) & & & & & & & & & & & \\
\hline & 18 & 53 & (46) & 53 & (46) & & & & & & & & & & & \\
\hline & 17 & 47 & (25) & 36 & (19) & 8 & (4) & 3 & (2) & & & & & 11 & (6) & 2.3 \\
\hline & & & & & & & & & & & & & & & (11 & \\
\hline mean & & 167 & (33) & 104 & (28) & 57 & (5) & 4 & (1) & 3 & (0) & & & 127 & ) & 6 \\
\hline $\max$ & & 534 & (46) & 292 & (46) & 221 & (15) & 11 & (2) & 10 & (1) & & & 242 & (16) & 11 \\
\hline $\min$ & & 33 & (25) & 33 & (19) & 0 & (0) & 0 & (0) & 0 & (0) & & & 11 & (6) & 2 \\
\hline
\end{tabular}

1

$2 \uparrow$ suspected error associated with determination of total P composition, site removed from subsequent analysis.

t Total $\mathrm{P}$ recovered by alkaline extraction

$\S$ Total phosphonates

II Total orthophosphate

$\dagger$ Total phosphomonoesters

t+ Ratio of total phosphomonoesters: total phosphodiesters 
Supplementary Table 4. Inorganic polyphosphates as determined by solution ${ }^{31} \mathrm{P}$ NMR spectroscopy of wetland soils. Values represent total inorganic polyphosphates delineated into pyrophosphate, and the terminal (TR) and mid-chain (MR) of long chain (n > 3)

polyphosphates.

\begin{tabular}{|c|c|c|c|c|c|}
\hline & \multicolumn{2}{|c|}{ Pyrophosphate } & \multicolumn{3}{|c|}{ Long chain polyphosphate } \\
\hline & \multirow[t]{2}{*}{$\mu \mathrm{g} \mathrm{g}^{-1}$} & \multirow[t]{2}{*}{$\%$ total P } & \multicolumn{2}{|c|}{$\mu \mathrm{g} \mathrm{g}^{-1}$} & \multirow[t]{2}{*}{$\%$ of total P } \\
\hline & & & TR & MR & \\
\hline \multicolumn{6}{|l|}{ Group A } \\
\hline 1 & 7.5 & 0.8 & trace & 35.5 & 3.6 \\
\hline 20 & 12.6 & 1.1 & trace & 110.1 & 9.8 \\
\hline 21 & 19.5 & 2.3 & trace & 98.9 & 11.6 \\
\hline 22 & 7.5 & 1.3 & trace & 71.6 & 12.4 \\
\hline 26 & 4.9 & 2.1 & 7.9 & 28.0 & 15.1 \\
\hline 27 & 4.3 & 1.2 & 6.2 & 27.3 & 9.4 \\
\hline mean & 9.4 & 1.5 & 2.4 & 61.9 & 10.3 \\
\hline \multicolumn{6}{|l|}{ Group B } \\
\hline 7 & 9.1 & 1.0 & 6.3 & 14.2 & 2.2 \\
\hline 8 & 5.4 & 0.7 & 7.0 & 37.6 & 6.0 \\
\hline 9 & 4.9 & 9.8 & - & - & \\
\hline 10 & 9.7 & 1.3 & 6.2 & 9.9 & 2.2 \\
\hline 11 & 4.3 & 0.5 & - & - & \\
\hline 12 & 5.6 & 0.5 & 5.6 & 13.6 & 1.8 \\
\hline 13 & 9.2 & 2.6 & - & - & \\
\hline 14 & 8.8 & 1.0 & - & - & \\
\hline mean & 7.1 & 2.2 & 3.9 & 9.4 & 1.5 \\
\hline \multicolumn{6}{|l|}{ Group C } \\
\hline 2 & 30.9 & 3.5 & 19.5 & 31.1 & 5.8 \\
\hline 3 & 41.6 & 4.4 & 25.9 & 5.9 & 3.4 \\
\hline 4 & 40.2 & 2.8 & 17.5 & 30.6 & 3.3 \\
\hline 5 & 24.6 & 2.5 & 12.1 & 35.2 & 4.8 \\
\hline 6 & 136.3 & 3.9 & 30.1 & 31.1 & 1.7 \\
\hline 15 & 32.3 & 2.7 & 10.6 & 6.8 & 1.5 \\
\hline 16 & 46.2 & 3.9 & 6.3 & 7.8 & 1.2 \\
\hline 23 & 6.6 & 2.4 & 1.7 & 3.1 & 1.7 \\
\hline 24 & 9.6 & 3.1 & - & - & \\
\hline 28 & 10.3 & 1.5 & 23.6 & 39.8 & 9.3 \\
\hline mean & 37.9 & 3.1 & 14.7 & 19.1 & 3.3 \\
\hline Group D & & No inorgan & olyphosph & s detected & \\
\hline
\end{tabular}


1 Supplementary Table 5. Correlation coefficients between microbial P (\% of total P) and P 2 forms determined by solution ${ }^{31} \mathrm{P}$ NMR spectroscopy (\% of total P).

3

\begin{tabular}{lrr}
\hline Phosphorus form & $\begin{array}{c}\text { Spearman rho } \\
\text { correlation }\end{array}$ & $p$ \\
\hline Phosphonate & -0.21 & $\mathrm{~ns}$ \\
Orthophosphate & -0.38 & $\mathrm{~ns}$ \\
Phosphomonoesters & -0.15 & $\mathrm{~ns}$ \\
DNA & 0.57 & $<0.01$ \\
Other phosphodiesters & 0.30 & $\mathrm{~ns}$ \\
Pyrophosphate & 0.38 & $\mathrm{~ns}$ \\
Long chain Polyphosphate & 0.80 & $<0.001$ \\
Residual & -0.05 & $\mathrm{~ns}$ \\
\hline
\end{tabular}

4 


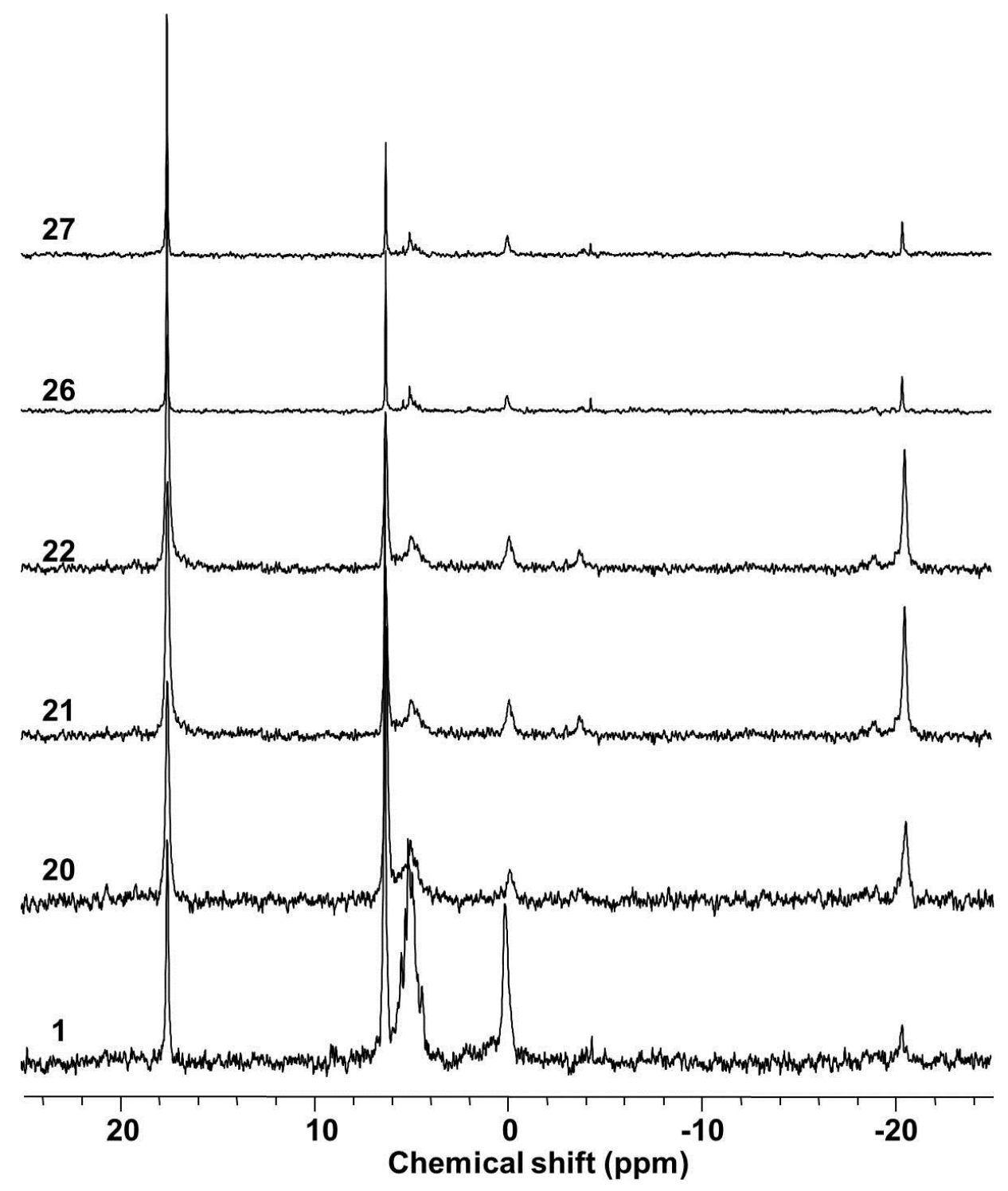

Supplementary Figure 1: Solution 31P NMR spectra of biogenic P composition within group A wetlands (high organic low pH). Spectra acquired using an Avance-500 (500.4 MHz 1H), Magnex 11.8 Tesla/54 mm Bore, at $\mathrm{pH}>13$ using a simple zgig pulse program and calibrated $30^{\circ}$ pulse angle. Spectra presented here using $15 \mathrm{~Hz}$ line broadening scaled and referenced to internal standard methylenediphosphonic acid $(\delta=17.46 \mathrm{ppm})$. 


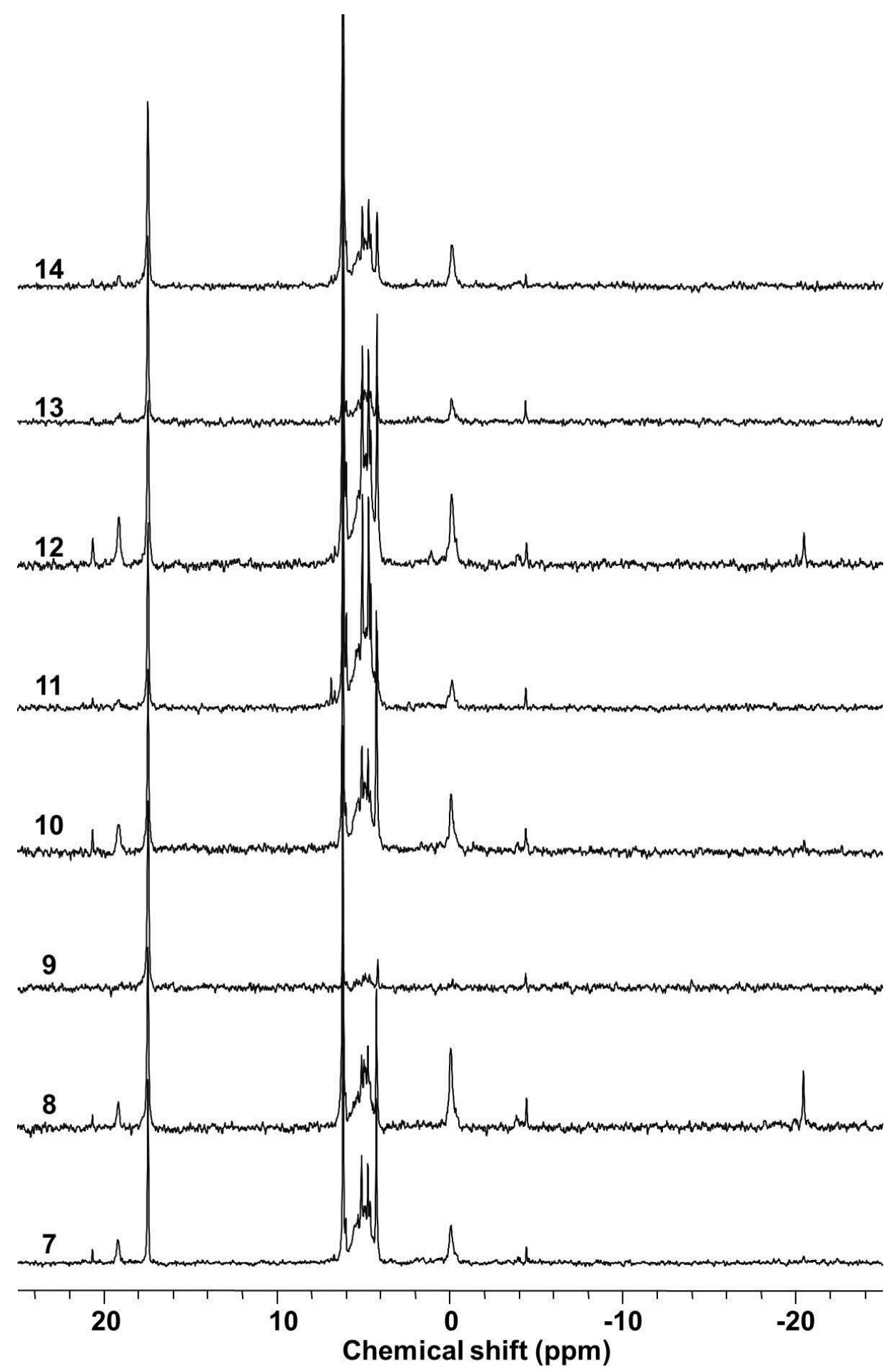

Supplementary Figure 2: Solution 31P NMR spectra of biogenic P composition within group B wetlands (low organic low pH). Spectra acquired using an Avance-500 (500.4 MHz 1H), Magnex 11.8 Tesla/54 mm Bore, at $\mathrm{pH}>13$ using a simple zgig pulse program and calibrated $30^{\circ}$ pulse angle. Spectra presented here using $15 \mathrm{~Hz}$ line broadening scaled and referenced to internal standard methylenediphosphonic acid $(\delta=17.46 \mathrm{ppm})$. 


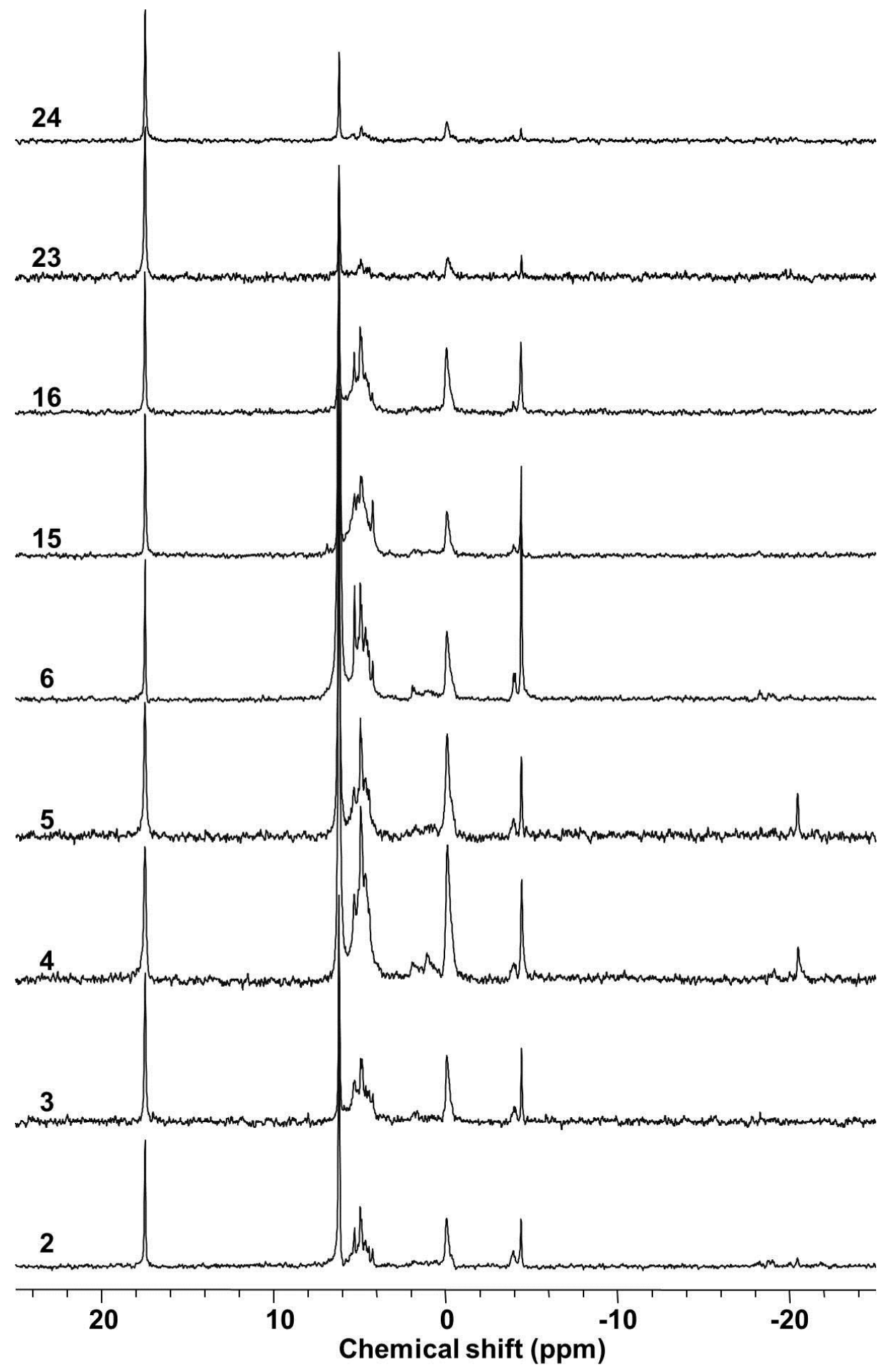

Supplementary Figure 3: Solution 31P NMR spectra of biogenic P composition within group C wetlands (high organic matter high $\mathrm{pH}$ ). Spectra acquired using an Avance-500 (500.4 MHz 1H), Magnex 11.8 Tesla/54 mm Bore, at $\mathrm{pH}>13$ using a simple zgig pulse program and calibrated $30^{\circ}$ pulse angle. Spectra presented here using $15 \mathrm{~Hz}$ line broadening scaled and referenced to internal standard methylenediphosphonic acid $(\delta=17.46 \mathrm{ppm})$. 


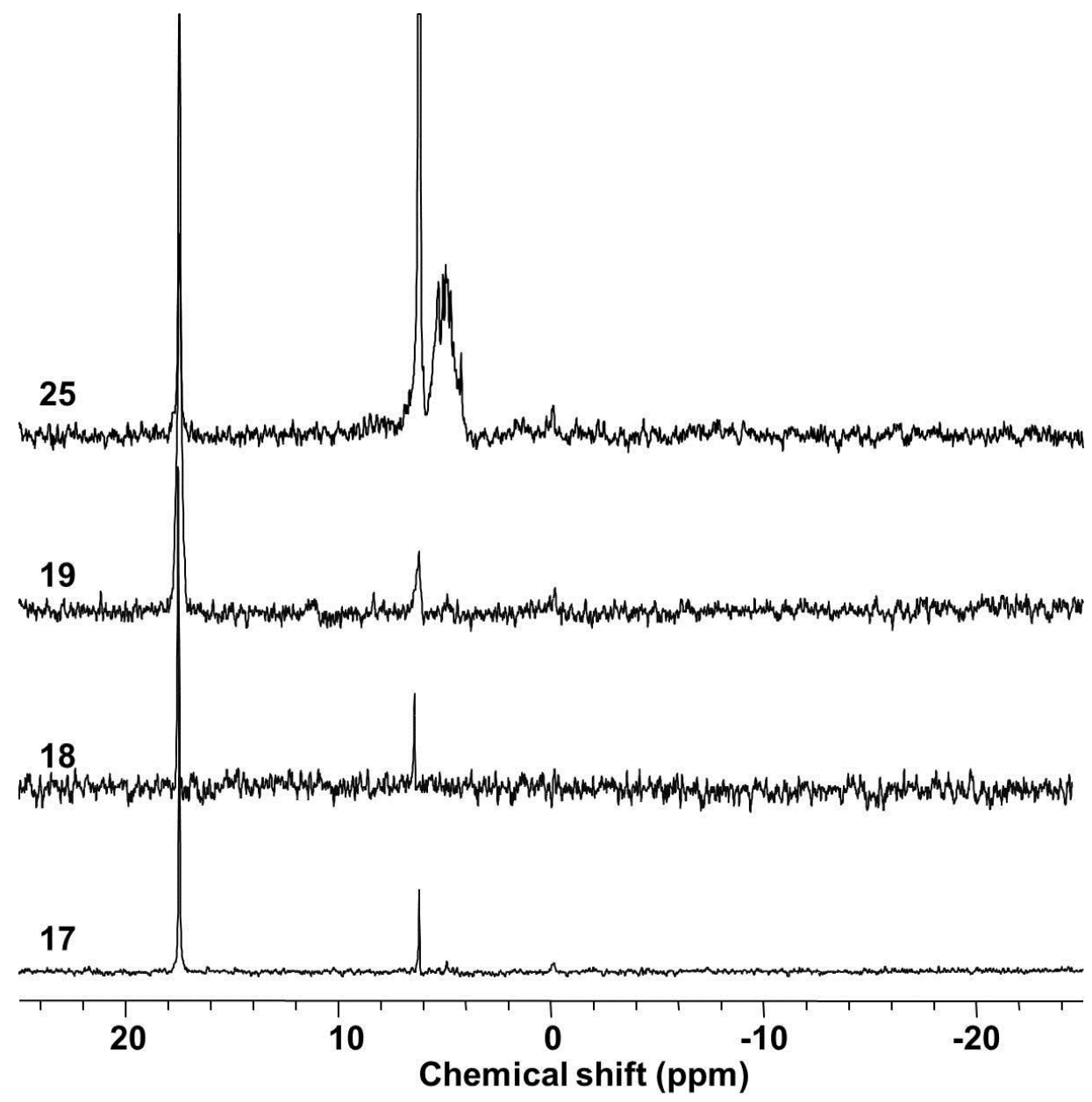

1

3 Supplementary Figure 4: Solution 31P NMR spectra of biogenic P composition within group

4 D wetlands (low organic matter high $\mathrm{pH}$ ). Spectra acquired using an Avance-500 (500.4 MHz

5 1H), Magnex 11.8 Tesla/54 mm Bore, at $\mathrm{pH}>13$ using a simple zgig pulse program and

6 calibrated $30^{\circ}$ pulse angle. Spectra presented here using $15 \mathrm{~Hz}$ line broadening scaled and

7 referenced to internal standard methylenediphosphonic acid ( $\delta=17.46 \mathrm{ppm})$. 